\title{
Resistance Patterns of Mycobacterium tuberculosis to First-Line Anti-TB Drugs in Kaduna State, North-Western Nigeria
}

\author{
${ }^{1}$ Aliyu, M. S., ${ }^{2}$ Garba, I., ${ }^{1}$ Tijjani, M. B., ${ }^{1}$ Doko, M. H.I., ${ }^{3}$ Mamuda, K., ${ }^{1}$ Suleiman, M. A. \\ and ${ }^{1}$ Hussaini, I. M. \\ ${ }^{1}$ Department of Microbiology, Faculty of Life Sciences, Ahmadu Bello University, Zaria \\ ${ }^{2}$ Department of Medical Microbiology, Faculty of Medical Laboratory Science, Usmanu Danfodiyo \\ University, Sokoto \\ ${ }^{3}$ National Tuberculosis and Leprosy Training Centre, Saye, Zaria
}

\begin{abstract}
The problem of tuberculosis (TB) in Nigeria has been made worse by the issues of drug resistant Mycobacterium tuberculosis. Resistance associated with treatment failures has become a common occurrence worldwide. Of particular concern is the increasing prevalence of multi drug resistant M. tuberculosis; organisms resistant to isoniazid and rifampicin, the two drugs that form the backbone of modern short-course therapy. Susceptibility of forty (40) M. tuberculosis isolates to isoniazid (INH), rifampicin (RIF), streptomycin (SM) and ethambutol (EMB) was determined by the proportion method on Lowenstein Jensen egg based slopes containing critical concentrations of INH, RIF, SM and EMB $(0.2 \mu \mathrm{g} / \mathrm{ml}, 40 \mu \mathrm{g} / \mathrm{ml}, 4.0 \mu \mathrm{g} / \mathrm{ml}$ and $2.0 \mu \mathrm{g} / \mathrm{ml}$ respectively). Overall drug resistance was found in $21(52.5 \%)$ isolates. Mono-drug resistance was seen in $9(22.5 \%)$ of the isolates tested and was against streptomycin. Two $(5.0 \%)$ isolates were found to be MDR. Five $(12.5 \%)$ were poly-drug resistant. One isolate showed poly-drug resistance to SM and INH, 2 (5\%) were poly-drug resistant to SM and EMB, 1 (2.5\%) was resistant to INH and EMB. Poly resistance to SM, INH and EMB was found in only 1 isolate. No poly-resistance to RIF and EMB, RIF and SM or RIF/SM/EMB was observed.

Key words: Resistance, Mycobacterium tuberculosis, first-line anti-TB drugs, Kaduna, Northwestern Nigeria
\end{abstract}

\section{INTRODUCTION}

Despite several international efforts, tuberculosis remains a leading infectious cause of death. The death rates, however, only partially depict the global TB threat; more than $80 \%$ of TB patients are in the economically productive age of 15 to 49 years (WHO, 2013). Global tuberculosis control efforts have been threatened by the emergence of multidrug resistant tuberculosis (MDR-TB). MDR-TB is defined as strains of Mycobacterium tuberculosis which show high level resistance to both isoniazid and rifampicin, with or without resistance to other anti TB drugs (WHO, 2013). MDR-TB is estimated to cause $4 \%$ of new tuberculosis cases in the developing world. Patients infected with MDR strains are not only difficult to cure but are also more likely to remain sources of infection for a longer period of time than those with drug susceptible organisms. MDR-TB requires longer duration of treatment (up to 2 years) to achieve cure, in comparison with the 6 month treatment for drug susceptible TB, lower cure rates and even higher default rates. The cost of drugs to treat an MDR-TB case can be up to 100 times more expensive than the cost of treating a drug susceptible TB case (Leimane and Leimans, 2006).

Understanding the scientific basis of short course 6 month chemotherapy for tuberculosis helps to explain why the loss of sensitivity to both isoniazid and rifampicin, even without resistance to additional drugs, has such major effects on outcome. Numerous controlled trials have shown that a 6 month regimen of rifampicin and isoniazid, supplemented by pyrazinamide and streptomycin or ethambutol for the first 2 months, will provide a cure in > 95\% of cases if the medication is taken correctly. Such a regimen also renders infectious cases non infectious in 2 weeks (Ormerod, 1997). Each drug varies in its ability to kill the tubercle bacilli (bactericidal ability), to deal with persistent organisms which are only occasionally metabolically active (sterilizing ability) and to prevent the emergence of drug resistance (Ormerod, 1997). Isoniazid is the best bactericidal drug and if mono resistance to this occurs, treatment with rifampicin and ethambutol has to be extended for 9 - 12 months, in addition to 2 months 
initial pyrazinamide. Rifampicin is the best sterilizing drug, and mono resistance to this drug requires treatment with isoniazid and ethambutol for 18 months, with 2 months initial pyrazinamide.

Therefore, loss of response to both the main bactericidal drug and the main sterilizing drug means that patients remain infectious for much longer time, both in the community and in hospital, that treatment is required for at least 12 and possibly more than 24 months, and that less effective and more toxic second line drugs have to be used (Joint Tuberculosis Committee of the British Thoracic Society, 1998). To the best of our knowledge, there is little information on the drug resistance patterns of M. tuberculosis in Kaduna State. This study was therefore aimed at determining the resistance patterns of $M$. tuberculosis to first-line anti-TB drugs in Kaduna, North-western Nigeria.

\section{MATERIALS AND METHODS \\ Test mycobacteria}

The test isolates of $M$. tuberculosis were obtained from National Tuberculosis and Leprosy Training Centre, Saye, Zaria, Nigeria. These were isolated at the Centre from samples obtained from the three senatorial districts of the State.

The isolates were confirmed by standard Microbiological techniques. They were morphologically characterized by making a smear and staining by the Ziehl-Neelsen procedure. Typical acid fast bacilli were further subjected to test according to the manufacturer's instructions by SD BIOLINE TB $\mathrm{Ag} \quad$ MPT64 Rapid; a rapid immunochromatographic identification test for the M. tuberculosis complex (MTBC) that uses mouse monoclonal anti-MPT64. The kit has sensitivity and specificity of $98.6 \%$ and $100 \%$ respectively. The test cassette consists of a sample pad, a gold conjugate pad, a nitrocellulose membrane and an absorbent pad. Mouse monoclonal anti-MPT64 was immobilized on the nitrocellulose membrane as the capture material (test line). Another antibody which recognized another epitope of MPT64, conjugated with colloidal gold particles was used for antigen capture and detection in a sandwich type assay.

Four colonies were suspended in $200 \mu \mathrm{l}$ of the extraction buffer prior to the test. The cassette was removed from the foil pouch and placed on a flat dry surface. One hundred micro litres $(100 \mu \mathrm{l})$ of the suspended colonies in buffer was added into the sample well. As the test began to run, a purple colour moved across the result window in the centre of the device. After 15 minutes of sample application, the appearance of two colour bands ("T" test band and " $C$ " control band) within the result window was considered a positive result. Confirmed MTBC isolates were stored at $-20^{\circ} \mathrm{C}$ for further use.

\section{Drug susceptibility testing}

Susceptibility to isoniazid (INH), rifampicin (RIF), streptomycin (SM) and ethambutol (EMB) was determined by the proportion method on Lowenstein Jensen egg based slopes containing different concentrations of INH, RIF, SM and EMB $(0.2 \mu \mathrm{g} / \mathrm{ml}, 40 \mu \mathrm{g} / \mathrm{ml}, 4.0 \mu \mathrm{g} / \mathrm{ml}$ and 2.0 $\mu \mathrm{g} / \mathrm{ml}$ respectively) (Canetti et al., 1969). Standard antibiotic powders (INH, RIF, SM and EMB) were obtained from Sigma-Aldrich (Lot. No. SLBC 3024V; SLBD 2314V; BCBK 3385V; SLBF 2556V respectively).

The inoculum was prepared by directly suspending colonies grown for approximately three weeks on Lowenstein Jensen drug free slopes to a turbidity equivalent to a 1.0 MacFarland standard. The 1.0 MacFarland standardised suspension was further diluted to $10^{-1}, 10^{-2}$ and $10^{-3}$. The $10^{-1}$ suspension was subsequently inoculated unto the drugcontaining medium. Three drug-free LJ slopes were inoculated with 1:10, 1:100 and 1:1000 diluted suspensions of a 1.0 MacFarland standardised inoculum. This was done for each sample tested. The drug-susceptible MTB reference strain ATCC 27294 (H37Rv) was used as a susceptible control and known resistant strains (ATCC 35825 H37Rv for INH and SM, ATCC35838 H37Rv for RIF, ATCC 35837 H37Rv for $\mathrm{EMB}$ ) were used as resistant controls. The slopes were incubated at $37^{\circ} \mathrm{C}$ and read after 4 and 6 weeks. An isolate was considered resistant if the proportion of bacilli resistant to the critical concentration of a drug exceeded 1\% (Canetti et al., 1963, Canetti et al., 1969).

\section{RESULTS}

Out of the $40 \mathrm{M}$. tuberculosis tested, 21 were found to be resistant to at least one first-line anti TB-drug giving a percentage resistance of $52.50 \%$ (Figure I). 


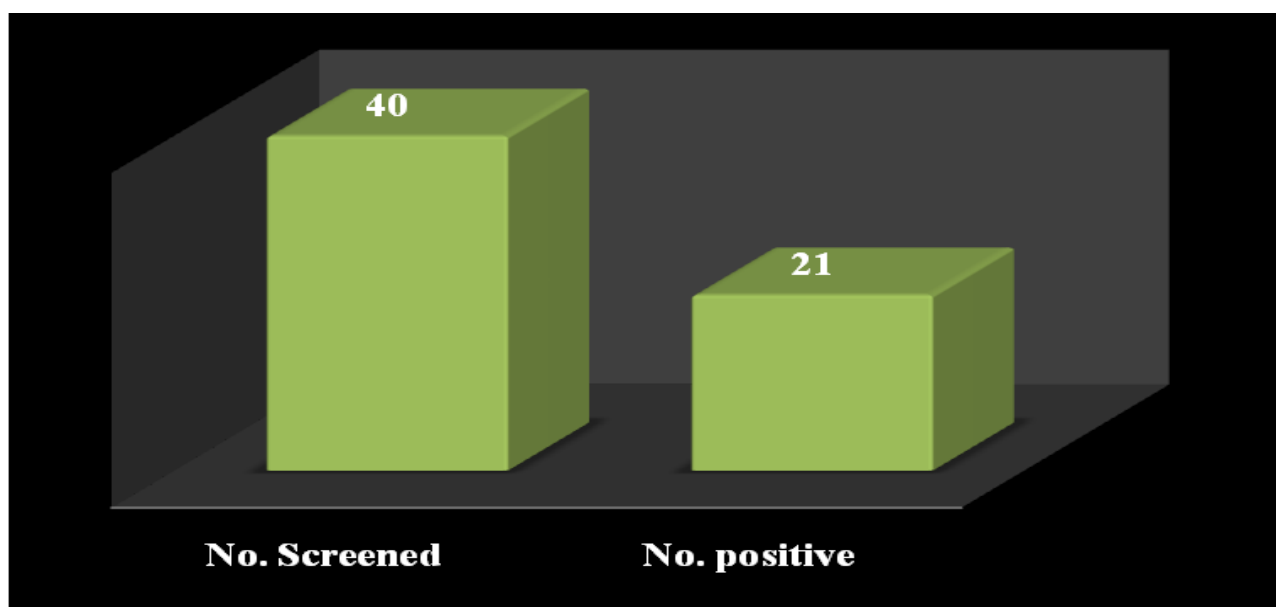

Figure I: Overall resistance of $M$. tuberculosis to first-line anti-TB drugs

Resistance pattern to any of the first-line anti-TB drugs is presented in Table 1 . Thirteen (32.5\%) of the isolates were resistant to SM, $3(7.5 \%)$ were resistant to INH, $2(2.5 \%)$ were resistant to RIF and resistance to EMB was found in $3(7.5 \%)$ isolates. Mono drug resistance was seen in $9(22.5 \%)$ of the isolates tested. All mono drug resistance was against streptomycin as shown in Table 2. Four $(10.0 \%)$ were poly drug resistant. Two isolates $(5.0 \%)$ showed poly-drug resistance to SM EMB, 1 (2.5\%) isolate was resistant to INH EMB. Poly resistance to SM INH EMB was found in only $1(2.5 \%)$ new case. No poly-resistance to RIF EMB, RIF SM or RIF SM EMB was observed.

Table 1: Any drug resistance pattern of $M$. tuberculosis to first-line anti-TB drugs

\begin{tabular}{lcc}
\hline Drug-resistance patterns & Frequency & \% Resistance \\
\hline SM & 13 & 32.5 \\
INH & 3 & 7.5 \\
RIF & 2 & 2.5 \\
EMB & 3 & 7.5 \\
Total & 21 & 52.5 \\
\hline
\end{tabular}

Key: Streptomycin (SM), isoniazid (INH), rifampicin (RIF), and ethambutol (EMB)

Table 2: Mono drug resistance pattern of $M$. tuberculosis to first-line anti-TB drugs

\begin{tabular}{lcc}
\hline Drug-resistance patterns & Frequency & \% Resistance \\
\hline SM & 9 & 22.5 \\
INH & 0 & 0.0 \\
RIF & 0 & 0.0 \\
EMB & 0 & 0.0 \\
Total & 9 & 22.5 \\
\hline
\end{tabular}

Key: Streptomycin (SM), isoniazid (INH), rifampicin (RIF), and ethambutol (EMB)

Table 3: Poly drug resistance pattern of $M$. tuberculosis to first-line anti-TB drugs

Drug-resistance patterns $\quad \mathrm{N}=\mathbf{4 0}$

\begin{tabular}{lcc} 
& Frequency & \% Resistance \\
\hline SM INH & 0 & 0.0 \\
SM EMB & 2 & 5.0 \\
INH EMB & 1 & 2.5 \\
RIF EMB & 0 & 0.0 \\
RIF SM & 0 & 0.0 \\
RIF SM EMB & 0 & 0.0 \\
SM INH EMB & 1 & 2.5 \\
Total & 4 & 10 \\
\hline
\end{tabular}

Key: Streptomycin (SM), isoniazid (INH), rifampicin (RIF), and ethambutol (EMB) 
Two (5.0\%) of the isolates were found to be multi drug resistant i.e. resistant to at least INH and RIF as presented in figure II.

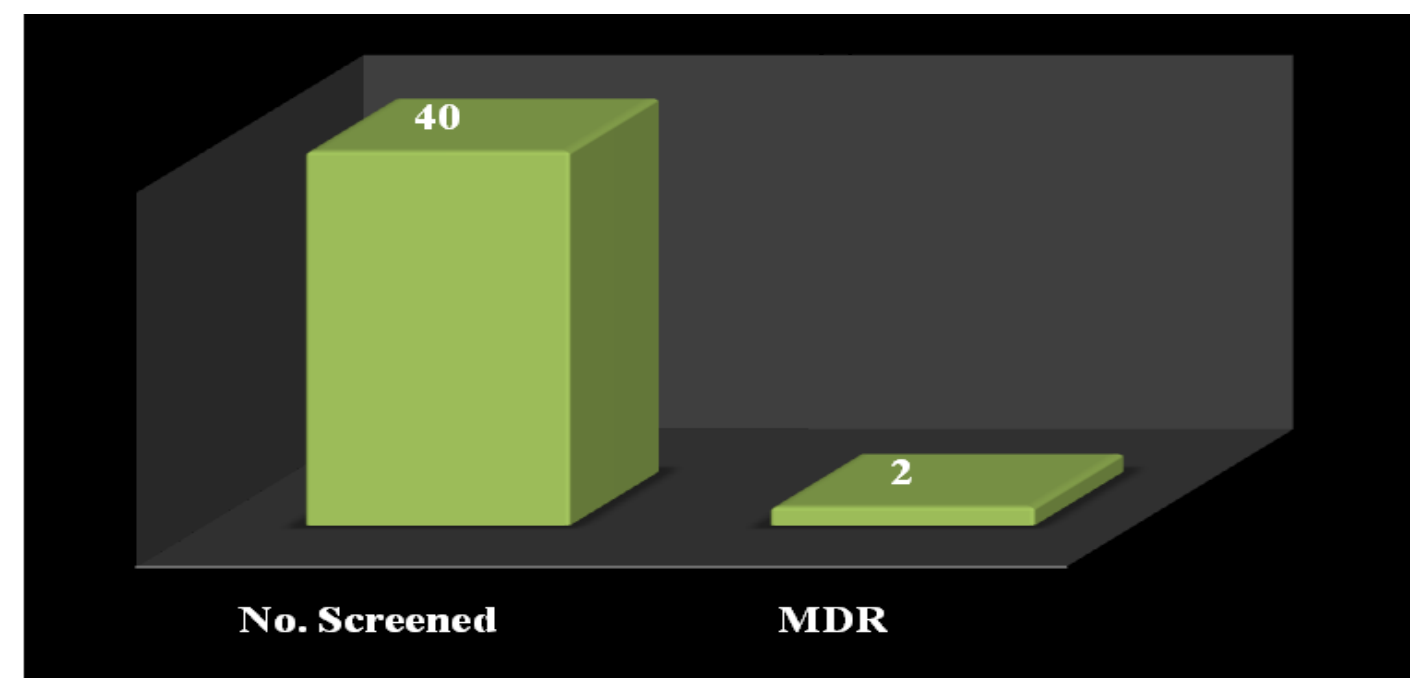

Figure II: Multi drug resistant $M$. tuberculosis

\section{DISCUSSION}

Drug-resistant TB ultimately develops from the inadequate treatment of active pulmonary TB. The resistance to first line anti-TB drugs in this study was found to be $52.5 \%$ among pulmonary TB patients in Kaduna State. This may result from poor prescription practices among medical doctors with poor drug selection and insufficient treatment duration as pointed out in earlier studies (Sharma and Mohan, 2006). Systemic problems, through inadequate public health resources and unpredictable drug supplies or supply of sub standard drugs, also play a role (Mukherjee et al., 2004).

Erratic or selective compliance to treatment and default among patients is another key factor as it causes M. tuberculosis to be exposed to sub-lethal doses for insufficient durations. These could thus, result in treatment failure and foster emergence of drug-resistant TB (Pablos-M'endez et al., 1997).

Mono-drug resistance was seen in the isolates tested and was against streptomycin. The highest resistance to streptomycin was not surprising because it is used for the treatment of other disease conditions such as brucellosis and cross-resistance with other aminoglycosides such as gentamicin which are commonly used in our settings for many years. Similar results of high resistance to streptomycin were previously reported; $30.6 \%$ in the national DR survey in Nigeria (National Drug-resistant TB prevalence survey report, Nigeria, 2012), 34\% in Sudan (Hassan et al., 2012) 18.8\% in Cameroon (Meriki et al., 2013) and $28.1 \%$ in India (Sethi et al., 2013).
The most important measure of TB drug resistance is the number of new cases that are MDR-TB (Dye et al., 2002). According to Onyedum et al. (2017), the prevalence of MDRTB in Nigeria among new and previously-treated cases was $6.0 \%$. This study showed a prevalence rate of $5.0 \%$. Drug resistance in $M$. tuberculosis occurs as a result of random spontaneous chromosomal mutations during natural cell replication. The availability of drugs on the open market and a private sector that delivers drugs to the population in an unregulated fashion in Nigeria could also be factors that might favour development of MDR-TB.

\section{CONCLUSION AND RECOMMENDATION}

The study showed an overall high rate of TB drug resistance $(52.5 \%$ any drug resistance; $12.5 \%$ poly-drug resistance and $22.5 \%$ Monodrug resistance). It also demonstrated that the prevalence of MDR-TB (5.0\%) is high in the State.

It is therefore, recommended that regular supply of standard drugs as well as strict adherence to the Directly Observed Therapy Short course strategy should be ensured by the authorities concerned, appropriate measures should be taken by the relevant authorities to prevent the indiscriminate and sub-therapeutic use of anti-TB drugs to avert the emergence of resistant strains, and improved health care facilities and standard of living as well as proper education of the public on TB drug resistance are also recommended. 


\section{REFERENCES}

Canetti, G., Froman, S., Grosset, J., Hauduroy, P., Langerova, M., Mahler, H.T., Meissner, G., Mitchison, D.A., and Sula, L. (1963). Mycobacteria: Laboratory methods for testing drug sensitivity and resistance. Bulletin of the World Health Organization, 29:565-578.

Canetti, G., Fox, W., Khomenko, A., Mahler, H.T., Menon, N.K., Mitchison, D.A., Rist, N., Smelev, N.A. (1969). Advances in techniques of testing mycobacterial drug sensitivity, and the use of sensitivity tests in tuberculosis control program. Bulletin of World Health Organization, 41(1):21-43.

Dye, C., Williams, B.G., Espinal, M.A., and Raviglione, M.C. (2002)."Erasing the world's slow stain: strategies to beat multi drug resistant tuberculosis," Science, 295(5562): 2042-2046.

Hassan, S.O., Musa, M.T., Elsheikh, H.M., Eleragi, A.M.S. and Saeed, N.S. (2012). Drug Resistance in Mycobacterium tuberculosis isolates from Northeastern Sudan. British Journal of Medicine and Medical Research, 2(3): 424-433.

Joint Tuberculosis Committee of the British Thoracic Society (1998). Chemotherapy and management of tuberculosis in the United Kingdom: recommendations. Thorax; 53, 536-548.

Leimane, V. and Leimans J. (2006). Tuberculosis control in Latvia: integrated DOTS and DOTS-Plus programmes. Eurosurveillance;11:1-3.

Meriki, H.D., Tufon, K.A., Atanga, P.N., AneAnyangwe, I.N., Anong, D.N., ChoNgwa, F. and Nkuo- Akenji, T. (2013). Drug Resistance Profiles of Mycobacterium tuberculosis Complex and Factors Associated with Drug Resistance in the Northwest and Southwest Regions of Cameroon. PLOS ONE,8(10): e77410.
Mukherjee, J.S., Rich, M.L., Socci, B.A., Joseph, A.R., Virú, K., Shin, F.A., Furin, S.S., Becerra, J.J., Barry, M.C., Kim, D.J., Bayona, J.Y., Farmer, J., Fawzi, P., and Seung, K.J. (2004). Programmes and principles for management of multidrug resistant tuberculosis. Lancet, 372(9407):474481.

National Drug-resistant TB prevalence survey report, Nigeria: National TB Drug resistance survey report (2012). Nigeria.

Onyedum, C. C., Alobu, I. and Ukwaja, K. N. (2017). Prevalence of drug-resistant tuberculosis in Nigeria: A systematic review and meta-analysis. PLoS ONE 12(7): e

Ormerod, L.P. (1997). Chemotherapy of tuberculosis. European Respiratory Journal, 2:273-297.

Pablos-M'endez, A., Knirsch, C.A., Barr, R.G., Lerner, B.H. and Frieden, T.R. (1997). Nonadherence in tuberculosis treatment: predictors and consequences in New York City. American Journal of Medicine, 102(2):164-170.

Sethi, S., Mewara, A., Dhatwalia, S.K., Singh, H., Yadav, R., Singh, K., Gupta, D., Wanchu, A. and Sharma, M. (2013). Prevalence of multidrug resistance in Mycobacterium tuberculosis isolates from HIV seropositive and seronegative patients with pulmonary tuberculosis in north India. Infectious Diseases, 13:137.

Sharma, S.K. and Mohan, A. (2006). Multidrugresistant tuberculosis: A menace that threatens to destabilize tuberculosis control. Chest, 130(1):261-272.

World Health Organization (2013).Global tuberculosis report 2013:http://www.who.int/tb/publicati ons/global_report/en/ 